\title{
ACKNOWLEDGMENTS
}

\section{A friend in need is a friend indeed: an appreciation letter to our 2020 reviewers and guest editors}

๑) Springer Nature Singapore Pte Ltd. 2021

Dear SEPR friends,

Your names are on our 2020 honor roll (see Table 1) because you are admired members of our SEPR community who offered generous and timely help when we were in desperate need in 2020. With your help, our community knowledge garden remained open and continued to harvest intellectual fruits in a world of lockdown, slowdown, and degrowth. ${ }^{1}$

The COVID-19 pandemic militates against human life but cannot erode humanistic spirit of helping one another. You have proved it with your compassionate, expeditious act.

We thank you and wish you and your loved ones a safe, healthy, and productive 2021 !

Wei-Ning Xiang, PhDs

The founding editor in chief (wxiang@uncc.edu)

On behalf of the SEPR editorial team

\footnotetext{
1 The metaphor "SEPR is a community knowledge garden" first appeared in "A tribute to our inaugural year reviewers" (The Editors of Socio-Ecological Practice Research 2020, p. 129)
} 
Table 1 The 2020 honor roll of SEPR reviewers and guest editors

\begin{tabular}{|c|c|c|c|}
\hline \multicolumn{4}{|c|}{ Our 2020 reviewers } \\
\hline Varenyam Achal & Ian Douglas & Stephen Kankam & Clare Palmer \\
\hline Kheir M Al-Kodmany & Cristian Echeverria & Susan Kemp & Michael Paolisso \\
\hline Christopher Auffrey & David Eisenhauer & Saehoon Kim & Hannah Partridge \\
\hline Elena Bakhanova & David Fielder & Natalie Klinard & Stephan Pauleit \\
\hline Ilaria Beretta & Paige Fischer & Julius Kotir & Alan Penn \\
\hline Ian Bishop & Courtney Flint & Praveen Kumar & Steven Porson \\
\hline Jessica Blythe & John Forester & Daniele La Rosa & Andrea Roberts \\
\hline Alec Brownlow & Hannah Fraser & Anita Lazurko & Rachel Scarlett \\
\hline M. Margaret Bryant & Christine Fürst & Dongying Li & Linda Shi \\
\hline J. Baird Callicott & Sara Gagne & Lu Liao & Jisoo Sim \\
\hline Nica Claudia Caló & Andrea K. Gerlak & Kuei-Hsien Liao & Hanna Sinare \\
\hline Deborah Carr & Grant Gilchrist & Katherine Lieberknecht & Rolanda Steenweg \\
\hline Jeffrey Chan & Leigh Graham & Theodore Chao Lim & Frederick Steiner \\
\hline Chundi Chen & Yvonne Gu & Jennifer NW Lim & John Troyer \\
\hline Tianyang Chen & Nathan Heavers & Greg Lindsey & Paul Van Auken \\
\hline Liding Chen & Shana Hirsch & Shiliang Liu & Sander van der Leeuw \\
\hline Eric Chu & Leah Hollstein & Yi Luo & Enrique Villacis Tapia \\
\hline Jessica Cockburn & Richard Hu & Marissa Matsler & Zhifang Wang \\
\hline Scott Colborne & Lingqian $\mathrm{Hu}$ & Benoit Mayer & Yuncai Wang \\
\hline Roberta Cucca & Tao Huang & Corina McKendry & Xinhao Wang \\
\hline KurtD Culbertson & Shu-Li Huang & George McKibbon & Runzi Wang \\
\hline Winifred Curran & Guoping Huang & Karen Murchie & Hui Wang \\
\hline Melissa Currie & Hilary Hughes & Vivian Nguyen & Oliver Wasonga \\
\hline Babu Dallakoti & Rebecca Jarvis & Holly Niner & Wei-Ning Xiang \\
\hline Tom Daniels & Wei JI & Barbara Norman & Bo Yang \\
\hline Catherine De Almeida & C.Y. Jim & Wikke Novalia & Fateme Zare \\
\hline Mina di Marino & Tim Johnson & Kwame Owusu-Daaku & Bo Zhang \\
\hline Jan Dick & Joseph Kangmennaang & Hannah Palko & Chun Zheng \\
\hline \multicolumn{4}{|c|}{ Our 2020 guest editors } \\
\hline Brett Bryan & Nica Claudia Caló & Lin Qi Feng & Curt Meine \\
\hline Enayat A. Moallemi & Forster Ndubisi & Martin Schultze & Haotian Zhong \\
\hline Praveen Kumar & Christine Fürst & & \\
\hline
\end{tabular}

\section{Reference}

The Editors of Socio-Ecological Practice Research (2020) A tribute to our inaugural year reviewers. Socio Ecol Pract Res 2(1):129-130. https://doi.org/10.1007/s42532-020-00041-8 